\title{
Nucleotide insertion and bypass synthesis of pyrene- and BODIPY-modified oligonucleotides by DNA polymerases $\dagger$
}

\author{
Claudia Wanninger-Weiß, ${ }^{a}$ Francesca Di Pasquale, ${ }^{b}$ Thomas Ehrenschwender, ${ }^{a}$ \\ Andreas Marx ${ }^{* b}$ and Hans-Achim Wagenknecht $* a$
}

The chromophores pyrene and bordipyrromethenylbenzene directly linked to the 5-position of uridine are tolerated and recognized as thymine derivatives by DNA polymerases in primer extension experiments.

If fluorophores are attached to DNA bases for oligonucleotide labeling, ${ }^{1}$ an alkyl chain linker is inserted between the chromophore and DNA base to allow the replication by DNA polymerases. However, the direct covalent attachment of chromophores to DNA bases yields unique optical properties, such as solvatochromism and exciplex-type emission ${ }^{2}$ that are suitable for DNA probing. A critical issue about this direct linkage is the question if the canonical base recognition complementarity persists in DNA polymerase-catalyzed primer extension experiments. ${ }^{3}$ For instance, fluorophore-labeled nucleosides and fluorosides can be applied as substrates for the DNA polymerase. ${ }^{4}$

Over the past years, we attached synthetically pyrene ${ }^{5-7}$ or ethynylpyrene $^{8}$, for example, to DNA bases for electron transfer studies and as fluorescent probes for DNA. To gain more insight into the counterbase selectivity, we performed primer extension experiments with a representative set of modified oligonucleotides (Scheme 1). The templates contained 5-(pyren-1-yl)-2'-deoxyuridine (1PydU), ${ }^{5}$ 5-(pyren-2yl)-2'-deoxyuridine (2PydU), ${ }^{7}$ 5-[4-(2,6-diethyl-4,4-difluoro1,3,5,7-tetramethyl-4-bora-3a-4a-diaza- $s$-indacyl)phenyl]-2'-deoxyuridine (BodU) or 8-(pyren-1-yl)-2'-deoxyguanosine $(\mathrm{PydG})^{6}$ as single modifications. The length of the radioactively labeled primer was chosen such that the modified nucleotide in the template strand codes for the first nucleotide during primer extension. Single-base incorporations were performed with each of the four dNTPs exclusively to get information about the insertion selectivity opposite to the modified nucleotide. In addition, experiments employing all four dNTPs simultaneously were performed to study the elongation bypassing the modification site.

\footnotetext{
${ }^{a}$ University of Regensburg, Institute for Organic Chemistry, D-93040 Regensburg, Germany.E-mail: achim.wagenknecht@chemie.uniregensburg.de; Fax: + 49 941-943-4617; Tel: + 49 941-943-4802

${ }^{b}$ University of Konstanz, Department of Chemistry, D-78457

Konstanz, Germany. E-mail: andreas.marx@uni-konstanz.de; Fax. + 49 7531-88-5140; Tel: + 49 7531-88-5139

$\dagger$ Electronic supplementary information (ESI) available: Synthesis, spectra and characterization details; primer extension experiments; DNA polymerase activity determination; UV absorption spectra. See
}

First we investigated the Klenow fragment (exo-) of E. coli DNA polymerase I (KF-) in its propensity to insert a nucleotide opposite the modified DNA nucleobase. Gel electrophoretic analysis of the radiometric primer extension reactions revealed that the canonical bases are predominantly incorporated, that means A opposite to $1 \mathrm{PydU}, 2 \mathrm{PydU}$ and BodU, and C opposite to PydG (Fig. 1). Only minor amounts of misincorporation of $\mathrm{G}$ opposite to $2 \mathrm{PydU}$ and less opposite to 1 PydU were observed. When all four dNTPs are present in the primer extension experiment, KF- is able to bypass all three types of uridine modifications (1PydU, 2PydU, and BodU) but not the modified guanosine $(\mathrm{PydG})$. This is a remarkable result since the steric hindrance by the chromophores, especially by the bordipyrromethenylphenyl substituent, was expected to be significant.

Subsequently, human DNA polymerase $\beta(\operatorname{Pol} \beta)$, a member of the DNA polymerase $\mathrm{X}$ family involved in DNA repair, and DNA polymerase Dpo4, a representative of the Y-family, were examined (Fig. 1). In the single nucleotide insertion experiments both enzymes placed the canonical nucleotides opposite the modification sites, but Pol $\beta$ was unable to incorporate any nucleotide opposite PydG. In contrast to KF-, a significant amount of misincorporation was not observed. However, both enzymes, Pol $\beta$ and Dpo4, were only able to bypass the modified uridines (except 1 PydU with Pol $\beta$ ) in experiments with all four dNTPs using higher polymerase concentrations and using an extended incubation time of $60 \mathrm{~min}$. Even under these conditions, PydG could not be bypassed by any of the polymerases (Fig. S4-S5, ESI $\dagger$ ). The reason for this might be that the pyrene at the 8-position induces the $s y n$-conformation ${ }^{5}$ of the nucleotide, yielding altered base pairing properties.

Since KF- was capable of bypassing DNA template modifications when all four dNTPs were present, we measured the activity of the enzyme on the respective templates in comparison to the unmodified template (Table 1). We employed an assay previously established to measure DNA polymerase activity on non-natural DNA primer template complexes. ${ }^{9}$ The data show that the chemical modifications significantly impair bypass efficiency. These effects are most pronounced when PydG was used. The $\mathrm{C} 5$ modifications at pyrimidines are somewhat better tolerated as has been observed with other modifications before. ${ }^{10}$

Finally, we examined the absorption (Fig. S1-S3, ESI $\dagger$ ) and fluorescence properties (Fig. 2) of the chromophore-uridine modified template-primer duplex in comparison with the synthetic full-length duplex. Additionally, an oligonucleotide was synthesized that contained the primer sequence and an additional $\mathrm{A}$ as counterbase to the chromophore-modified 

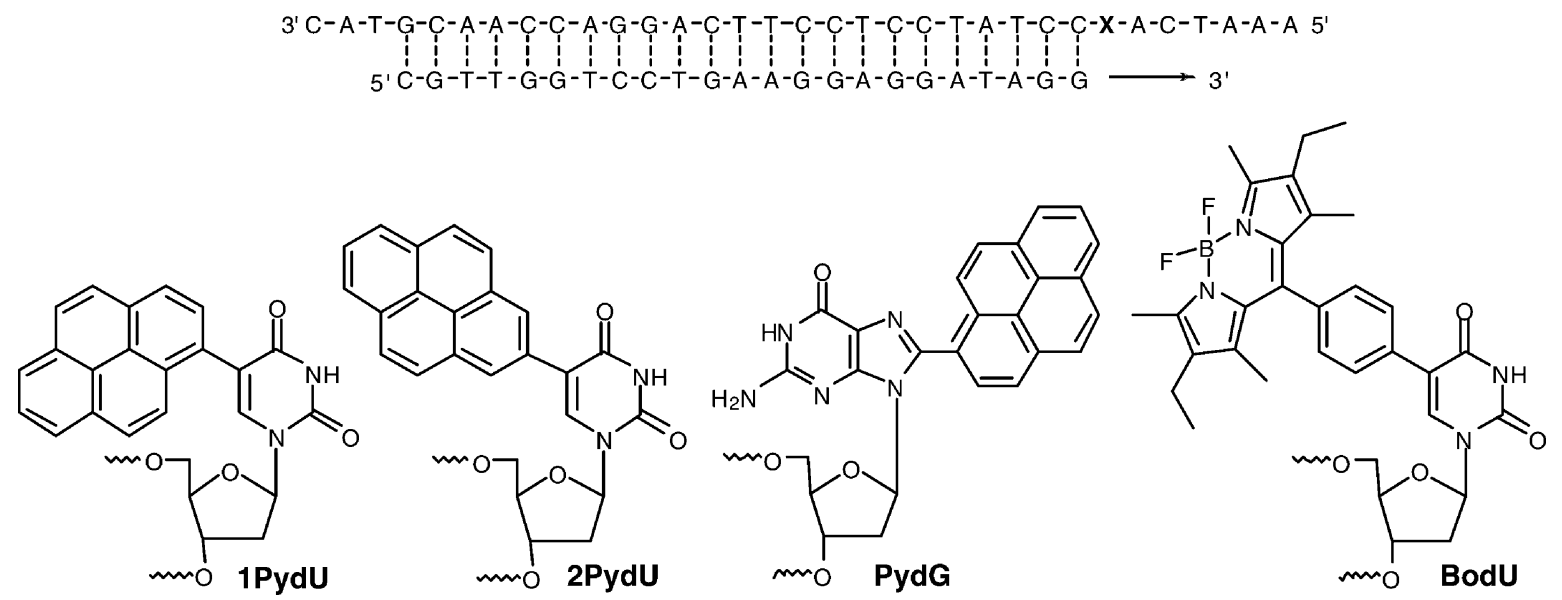

Scheme 1 DNA template and primer sequence (X = 1PydU, 2PydU, PydG, BodU).

uridine. Remarkably, with both 1 PydU and 2 PydU the emissions increase from the template-primer duplex to the fulllength duplex. Interestingly, elongation with one A opposite to 1 PydU or $2 \mathrm{PydU}$ is not sufficient to obtain this fluorescence enhancement. The BodU modification behaves completely independently of whether a primer extension has occurred or not. For all three modifications, the observed fluorescence

A

$30 \mathrm{nt}$

$3 \mathrm{nt}$
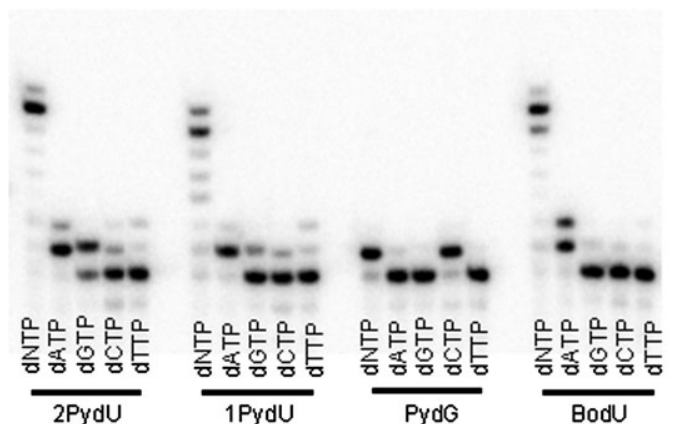

B

$23 \mathrm{nt}$
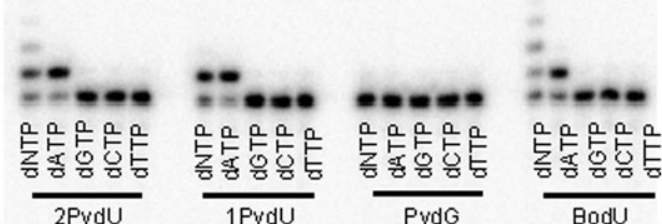

1Pydu

PydG

BodU

\section{C}

$30 \mathrm{nt}$

$23 \mathrm{nt}$
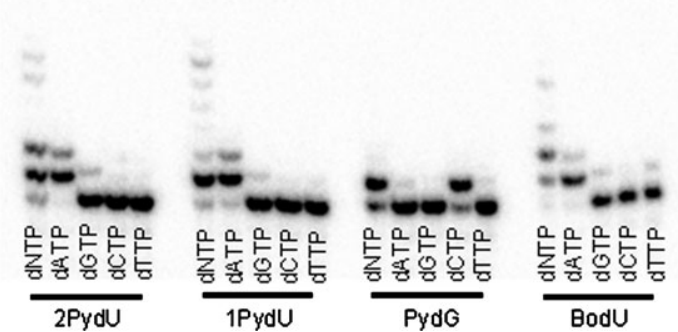

Fig. 1 Gel electrophoretic analysis of primer extension experiments with 1PydU-, 2PydU-, BodU- and PydG-modified templates: (A): Klenow fragment (exo-) of $E$. coli DNA polymerase I, (B) human DNA polymerase $\beta,(C)$ Dpo4 DNA polymerase. All reactions contained $200 \mu \mathrm{M}$ of the respective dNTP and were incubated for $30 \mathrm{~min}$ at $37{ }^{\circ} \mathrm{C}$. For more experimental details see the ESI†.
Table 1 Activities of KF- on unmodified and modified templates ${ }^{a}$

\begin{tabular}{ll}
\hline Oligonucleotide & [fmol dNTP incorporation per fmol pol per min] \\
\hline Non-modified & $11660 \pm 722.4$ \\
2PydU & $1395 \pm 71.8$ \\
1PydU & $457.5 \pm 17.3$ \\
PydG & $146.2 \pm 6.6$ \\
BodU & $1402 \pm 78.5$
\end{tabular}

${ }^{a}$ Data were obtained from multiple individual measurements. Instead of the modified building block a dA residue was present in the template strand. pol $=$ DNA polymerase.

changes are accompanied by changes in the emission maxima. This is typical for solvatochromic fluorophores. The exciplextype emission reacts to changes in the stacking situation from the single-strand to the full-length duplex.

In summary, we have shown that the DNA polymerasecatalyzed nucleotide incorporation opposite to attached pyrene and bordipyrromethenylbenzene at the 5-position of uridine follows Watson-Crick selectivity. KF- is also able to bypass the modification site during further elongation. These observations make these kinds of fluorescent labels promising tools for in vivo experiments in cell biology.

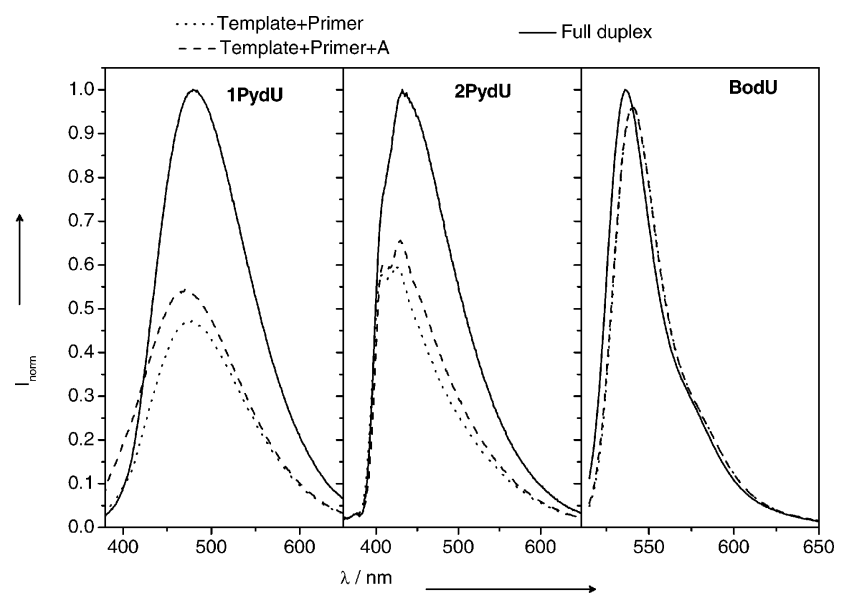

Fig. 2 Fluorescence spectra of $2.5 \mu \mathrm{M}$ duplex in $10 \mathrm{mM} \mathrm{Na}-\mathrm{P}_{\mathrm{i}}$-buffer, $\mathrm{pH} 7,250 \mathrm{mM} \mathrm{NaCl}, 20^{\circ} \mathrm{C}$, excitation at $360 \mathrm{~nm}$ (1PydU), $340 \mathrm{~nm}$ (2PydU), $510 \mathrm{~nm}$ (BodU). 


\section{Notes and references}

1 (a) See reviews and references therein: C. Wojczewski, K. Stolze and J. W. Engels, Synlett, 1999, 1667; (b) R. T. Ranasinghe and T. Brown, Chem. Commun., 2005, 5487; (c) A. Waggoner, Curr. Opin. Chem. Biol., 2006, 10, 62; (d) A. J. A. Cobb, Org. Biomol. Chem., 2007, 5, 3260.

2 Y. Wang, O. Haze, J. P. Dinnocenzo, S. Farid, R. S. Farid and I. R. Gould, J. Org. Chem., 2007, 72, 6970.

3 (a) E. T. Kool, Annu. Rev. Biochem., 2002, 71, 191; (b) A. A. Henry and F. E. Romesberg, Curr. Opin. Biotechnol., 2005, 16, 370.

4 (a) L. H. Thoresen, G.-S. Jiao, W. C. Haaland, M. L. Metzker and K. Burgess, Chem.-Eur. J., 2003, 9, 4603; (b) Y. Cho and E. T. Kool, ChemBioChem, 2006, 7, 669.

5 (a) E. Mayer, L. Valis, R. Huber, N. Amann and H.-A. Wagenknecht, Synthesis, 2003, 2335; (b) P. Kaden, E. Mayer-Enthart, A. Trifonov, T. Fiebig and H.-A. Wagenknecht, Angew. Chem., Int. Ed., 2005, 44, 1636.
6 L. Valis, E. Mayer-Enthart and H.-A. Wagenknecht, Bioorg. Med. Chem. Lett., 2006, 16, 3184.

7 C. Wanninger-Weiß and H.-A. Wagenknecht, Eur. J. Org. Chem., 2008, 64.

8 (a) E. Mayer, L. Valis, C. Wagner, M. Rist, N. Amann and H.-A. Wagenknecht, ChemBioChem, 2004, 5, 865; (b) C. Wagner, M. Rist, E. Mayer-Enthart and H.-A. Wagenknecht, Org. Biomol. Chem., 2005, 3, 2062.

9 (a) C. Gloeckner, K. B. M. Sauter and A. Marx, Angew. Chem., Int. Ed., 2007, 46, 3115; (b) N. Z. Rudinger, R. Kranaster and A. Marx, Chem. Biol., 2007, 14, 185; (c) K. B. M. Sauter and A. Marx, Angew. Chem., Int. Ed., 2006, 45, 7633.

10 (a) P. Čapek, H. Cahová, R. Pohl, M. Hocek, C. Gloeckner and A. Marx, Chem.-Eur. J., 2007, 13, 6115; (b) S. Jäger, G. Rasched, H. Kornreich-Leshem, M. Engeser, O. Thum and M. Famulok, $J$. Am. Chem. Soc., 2005, 127, 15071. 